\title{
Asymptotic estimates on the time derivative of entropy on a Riemannian manifold
}

\author{
Adrian P. C. $\operatorname{Lim}^{a}$, Dejun Luo ${ }^{a, b *}$ \\ ${ }^{a}$ UR Mathématiques, Université de Luxembourg, 6, rue Richard Coudenhove-Kalergi, L-1359 Luxembourg \\ ${ }^{b}$ Key Lab of Random Complex Structures and Data Science, Academy of Mathematics and \\ Systems Science, Chinese Academy of Sciences, Beijing 100190, China
}

\begin{abstract}
We consider the entropy of the solution to the heat equation on a Riemannian manifold. When the manifold is compact, we provide two estimates on the rate of change of the entropy in terms of the lower bound on the Ricci curvature and the spectral gap respectively. Our explicit computation for the three dimensional hyperbolic space shows that the time derivative of the entropy is asymptotically bounded by two positive constants.
\end{abstract}

MSC 2010: 58J05

Keywords: Heat equation, entropy, Ricci curvature, three dimensional hyperbolic space

\section{Introduction}

In Perelman's solution to the Poincaré conjecture, the $W$-functional (i.e. the entropy of the heat kernel) played an important role. Since then, there have been many attempts to understand or generalize this functional to other situations, see $[3,7,8,9]$. This work is motivated by $[8,9]$, where the author presented the expression for the time derivative of the entropy and studied its properties. Our aim is to estimate the asymptotic behavior of the time derivative of the entropy.

Let $M$ be a closed manifold, with dimension $n$. Equip $M$ with a Riemannian metric $g$ and define the corresponding Laplacian $\Delta \equiv \Delta_{g}$. We consider the following heat equation

$$
\frac{\partial}{\partial t} u=\frac{1}{2} \Delta u, \quad u_{0}=f
$$

where $f \in C^{1}(M,(0, \infty))$. In this work we follow the probabilists' convention (e.g. [1, 5]) of considering $\frac{1}{2} \Delta$ instead of the Laplacian $\Delta$. Let $\mathrm{d} x$ be the volume measure on $M$. Without loss of generality, we assume that $M$ has volume 1. If $\int_{M} f \mathrm{~d} x=1$, then $\int_{M} u_{t} \mathrm{~d} x \equiv 1$ for all $t>0$. Hence

$$
\int_{M} \frac{\partial}{\partial t} u_{t} \mathrm{~d} x=\frac{\mathrm{d}}{\mathrm{d} t} \int_{M} u_{t} \mathrm{~d} x=0
$$

Define the entropy

$$
\operatorname{Ent}\left(u_{t}\right)=-\int_{M} u_{t} \log u_{t} \mathrm{~d} x
$$

\footnotetext{
*Email: luodj@amss.ac.cn
} 
It is easy to see that $\operatorname{Ent}\left(u_{t}\right) \leq 0$ for all $t \geq 0$, and the equality holds if and only if $f \equiv 1$. By the integration by parts formula, it is easy to show that

$$
\frac{\mathrm{d}}{\mathrm{d} t} \operatorname{Ent}\left(u_{t}\right)=\frac{1}{2} \int_{M} \frac{\left|\nabla u_{t}\right|^{2}}{u_{t}} \mathrm{~d} x
$$

From this, we see that if the initial value $f$ is not constant, then $\operatorname{Ent}\left(u_{t}\right)$ is an increasing function of $t>0$. Denote by Ric the Ricci curvature tensor on $M$. The main result of this paper is

Theorem 1.1. Assume that there is a $k \in \mathbb{R}$ such that $\mathrm{Ric} \geq k \mathrm{Id}$. Then

$$
\frac{\mathrm{d}}{\mathrm{d} t} \operatorname{Ent}\left(u_{t}\right) \leq \begin{cases}\frac{e^{-k t}}{2}\left[\frac{1}{q_{0}}-\frac{e^{-k t}-1}{n k}\right]^{-1}, & k \neq 0 \\ \frac{n q_{0}}{2\left(n+q_{0} t\right)}, & k=0\end{cases}
$$

where $q_{0}=\int_{M} \frac{|\nabla f|^{2}}{f} \mathrm{~d} x$.

This result will be proved in Section 2. We conclude from Theorem 1.1 that if the Ricci curvature is bounded from below by a positive constant $k>0$, then the time derivative of the entropy has an exponential decay as $t \rightarrow \infty$; however, if $k<0$, then the time derivative of the entropy is asymptotically dominated by $-n k / 2>0$ as $t \rightarrow \infty$. The proof is based on the integration by parts formula and the inequality (2.14). For a compact Riemannian manifold with negative Ricci curvature, the estimate given in Theorem 1.1 is not satisfactory. In Section 3 we will give an estimate on the rate of change of entropy in terms of the spectral gap, which implies that the time derivative of the entropy always decrease exponentially to 0 , even though Ricci is bounded below by a negative constant (see Proposition 3.1).

One might wonder if a similar result holds when the manifold $M$ is non-compact. The difficulty in this case is the justification of the existence of the entropy and the integration by parts formula. According to [7, p.25], when the Ricci curvature of a non-compact manifold is nonnegative, the entropy formula has been established rigorously, see [3]. Therefore by Li-Yau's gradient estimate for positive solutions of the heat equation (1.1), we can show that the time derivative of the entropy does not exceed $n /(2 t)$, i.e. the decay rate of the entropy of heat kernel on $\mathbb{R}^{n}$; moreover, the time derivative achieves the critical value $n /(2 t)$ if and only if $M$ is isometric to $\mathbb{R}^{n}$, see Theorem 2.6. However, when $M$ has negative Ricci curvature, the situation is different. In the case of the three dimensional hyperbolic space $\mathbb{H}^{3}$, we will prove in Theorem 4.1 that the rate of change of entropy is asymptotically bounded by two positive constants.

\section{Rate of change of the entropy under curvature condition}

In this section we will provide the proof of Theorem 1.1. In fact we can deal with more general cases. To this end, let $Z$ be a $C^{1}$-vector field on $M$ and consider the second order differential operator

$$
L=\frac{1}{2} \Delta+Z
$$

Proposition 2.1. Let $f \in C^{1}(M,(0, \infty))$ and $u:[0, \infty) \times M \rightarrow \mathbb{R}_{+}$be a solution to the heat equation

$$
\frac{\partial}{\partial t} u=L u, \quad u_{0}=f
$$

We have

$$
\left(L-\frac{\partial}{\partial t}\right)\left(\frac{|\nabla u|^{2}}{u}\right)=\frac{1}{u}\left|\operatorname{Hess} u-\frac{\nabla u \otimes \nabla u}{u}\right|^{2}+\frac{1}{u}\left(\operatorname{Ric}(\nabla u, \nabla u)-2\left\langle D_{\nabla u} Z, \nabla u\right\rangle\right) .
$$


Proof. By the Weitzenböck formula,

$$
\frac{1}{2} \Delta\left(|\nabla u|^{2}\right)=\langle\nabla \Delta u, \nabla u\rangle+|\operatorname{Hess} u|_{H S}^{2}+\operatorname{Ric}(\nabla u, \nabla u)
$$

where Hess $u$ is the Hessian of $u$ and $|\cdot|_{H S}$ is the Hilbert-Schmidt norm. Next taking $Y=\nabla u$ and $g=u$ in Lemma 2.2 below gives us

$$
\langle\nabla u, \nabla Z(u)\rangle=\left\langle D_{\nabla u} Z, \nabla u\right\rangle+\left\langle\nabla u, D_{Z} \nabla u\right\rangle
$$

where $D$ is the Levi-Civita connection. As a result,

$$
Z\left(|\nabla u|^{2}\right)=Z\langle\nabla u, \nabla u\rangle=2\left\langle D_{Z} \nabla u, \nabla u\right\rangle=2\left\langle\nabla Z(u)-D_{\nabla u} Z, \nabla u\right\rangle .
$$

Finally $\frac{\partial}{\partial t}\left(|\nabla u|^{2}\right)=2\left\langle\frac{\partial}{\partial t}(\nabla u), \nabla u\right\rangle=2\left\langle\nabla\left(\frac{\partial}{\partial t} u\right), \nabla u\right\rangle$. Combining this with (2.2) and (2.3), and using the equation (2.1), we get

$$
\left(L-\frac{\partial}{\partial t}\right)\left(|\nabla u|^{2}\right)=|\operatorname{Hess} u|_{H S}^{2}+\operatorname{Ric}(\nabla u, \nabla u)-2\left\langle D_{\nabla u} Z, \nabla u\right\rangle .
$$

Now notice that

$$
\Delta\left(\frac{|\nabla u|^{2}}{u}\right)=u^{-1} \Delta\left(|\nabla u|^{2}\right)+|\nabla u|^{2} \Delta\left(u^{-1}\right)+2\left\langle\nabla u^{-1}, \nabla\left(|\nabla u|^{2}\right)\right\rangle .
$$

We have

$$
\Delta\left(u^{-1}\right)=-\frac{\Delta u}{u^{2}}+\frac{2}{u^{3}}|\nabla u|^{2}
$$

and

$$
\left\langle\nabla u^{-1}, \nabla\left(|\nabla u|^{2}\right)\right\rangle=-\frac{1}{u^{2}}\left\langle\nabla u, \nabla\left(|\nabla u|^{2}\right)\right\rangle=-\frac{2}{u^{2}}\left\langle D_{\nabla u} \nabla u, \nabla u\right\rangle=-\frac{2}{u^{2}}(\operatorname{Hess} u)(\nabla u, \nabla u) .
$$

Substituting the above equalities into (2.5) gives rise to

$$
\Delta\left(\frac{|\nabla u|^{2}}{u}\right)=\frac{1}{u} \Delta\left(|\nabla u|^{2}\right)-\frac{|\nabla u|^{2}}{u^{2}} \Delta u+\frac{2}{u^{3}}|\nabla u|^{4}-\frac{4}{u^{2}}(\operatorname{Hess} u)(\nabla u, \nabla u) .
$$

We also have

$$
\begin{aligned}
Z\left(\frac{|\nabla u|^{2}}{u}\right) & =\left\langle Z,|\nabla u|^{2} \nabla\left(u^{-1}\right)+u^{-1} \nabla\left(|\nabla u|^{2}\right)\right\rangle \\
& =-\frac{|\nabla u|^{2}}{u^{2}} Z(u)+\frac{1}{u} Z\left(|\nabla u|^{2}\right)
\end{aligned}
$$

and

$$
\frac{\partial}{\partial t}\left(\frac{|\nabla u|^{2}}{u}\right)=-\frac{|\nabla u|^{2}}{u^{2}} \frac{\partial}{\partial t} u+\frac{1}{u} \frac{\partial}{\partial t}\left(|\nabla u|^{2}\right)
$$

Consequently, by (2.6)-(2.8),

$$
\begin{aligned}
\left(L-\frac{\partial}{\partial t}\right)\left(\frac{|\nabla u|^{2}}{u}\right)= & \frac{1}{u}\left(L-\frac{\partial}{\partial t}\right)\left(|\nabla u|^{2}\right)-\frac{|\nabla u|^{2}}{u^{2}}\left(L-\frac{\partial}{\partial t}\right) u \\
& +\frac{|\nabla u|^{4}}{u^{3}}-\frac{2}{u^{2}}(\operatorname{Hess} u)(\nabla u, \nabla u),
\end{aligned}
$$


which, by (2.4), is equal to

$$
\begin{aligned}
& \frac{1}{u}\left(|\operatorname{Hess} u|_{H S}^{2}+\operatorname{Ric}(\nabla u, \nabla u)-2\left\langle D_{\nabla u} Z, \nabla u\right\rangle\right)+\frac{|\nabla u|^{4}}{u^{3}}-\frac{2}{u^{2}}(\operatorname{Hess} u)(\nabla u, \nabla u) \\
= & \frac{1}{u}\left(\left|\operatorname{Hess} u-\frac{\nabla u \otimes \nabla u}{u}\right|_{H S}^{2}+\operatorname{Ric}(\nabla u, \nabla u)-2\left\langle D_{\nabla u} Z, \nabla u\right\rangle\right) .
\end{aligned}
$$

The proof is complete.

Lemma 2.2. Let $Y, Z$ be two $C^{1}$-vector fields on $M$ and $g \in C^{2}(M)$. We have

$$
\langle Y, \nabla Z(g)\rangle=\left\langle D_{Y} Z, \nabla g\right\rangle+\left\langle Y, D_{Z} \nabla g\right\rangle .
$$

Proof. By the consistency of the Levi-Civita connection $D$ with the metric,

$$
\langle Y, \nabla Z(g)\rangle=Y[Z(g)]=Y\langle Z, \nabla g\rangle=\left\langle D_{Y} Z, \nabla g\right\rangle+\left\langle Z, D_{Y} \nabla g\right\rangle .
$$

Notice that

$$
\left\langle Z, D_{Y} \nabla g\right\rangle=(\operatorname{Hess} g)(Y, Z)=(\operatorname{Hess} g)(Z, Y)=\left\langle Y, D_{Z} \nabla g\right\rangle .
$$

The result follows.

Now we take $V \in C^{2}(M)$ and let $Z=\nabla V$. That is, we consider the heat equation

$$
\frac{\partial}{\partial t} u=L u=\frac{1}{2} \Delta u+\langle\nabla V, \nabla u\rangle, \quad u_{0}=f>0 .
$$

Define the measure $\mathrm{d} \mu=e^{2 V} \mathrm{~d} x$ on $M$, where $\mathrm{d} x$ is the volume element of $M$. Normalize the measure $\mu$ if necessary, we will assume that $\mu(M)=1$. For any $f, g \in C^{1}(M)$, the following integration by parts formula holds:

$$
-\int_{M} L f \cdot g \mathrm{~d} \mu=\frac{1}{2} \int_{M}\langle\nabla f, \nabla g\rangle \mathrm{d} \mu .
$$

Assume that $\mu(f)=\int_{M} f \mathrm{~d} \mu=1$. Then we have $\int_{M} u_{t} \mathrm{~d} \mu \equiv 1$ for all $t>0$. Hence

$$
\int_{M} \frac{\partial}{\partial t} u_{t} \mathrm{~d} \mu=\frac{\mathrm{d}}{\mathrm{d} t} \int_{M} u_{t} \mathrm{~d} \mu=0
$$

Define the entropy

$$
\operatorname{Ent}\left(u_{t}\right)=-\int_{M} u_{t} \log u_{t} \mathrm{~d} \mu .
$$

It is easy to see that $\operatorname{Ent}\left(u_{t}\right) \leq 0$ for all $t \geq 0$, and the equality holds if and only if $f \equiv 1$. Using the integration by parts formula $(2.10)$,

$$
\begin{aligned}
\frac{\mathrm{d}}{\mathrm{d} t} \operatorname{Ent}\left(u_{t}\right) & =-\int_{M}\left(\left(\log u_{t}\right) \frac{\partial}{\partial t} u_{t}+\frac{\partial}{\partial t} u_{t}\right) \mathrm{d} \mu=-\int_{M}\left(\log u_{t}\right) L u_{t} \mathrm{~d} \mu \\
& =\frac{1}{2} \int_{M}\left\langle\nabla \log u_{t}, \nabla u_{t}\right\rangle \mathrm{d} \mu=\frac{1}{2} \int_{M} \frac{\left|\nabla u_{t}\right|^{2}}{u_{t}} \mathrm{~d} \mu .
\end{aligned}
$$

From this, we see that if the initial value $f$ is not a constant, then $\operatorname{Ent}\left(u_{t}\right)$ is an increasing function of $t>0$.

We want to estimate the rate of change of the entropy, i.e. $\frac{\mathrm{d}}{\mathrm{d} t} \operatorname{Ent}\left(u_{t}\right)$, as $t \rightarrow \infty$. To this end, we will make use of the equality proved in Proposition 2.1. It is easy to see that

$$
\left|\operatorname{Hess} u-\frac{\nabla u \otimes \nabla u}{u}\right|_{H S}^{2}=u^{2}|\operatorname{Hess}(\log u)|_{H S}^{2} \geq \frac{u^{2}}{n}|\Delta(\log u)|^{2},
$$


where $n$ is the dimension of $M$, and the equality holds if and only if Hess $(\log u)=g$ Id with some function $g: \mathbb{R}_{+} \times M \rightarrow \mathbb{R}$. Therefore by Proposition 2.1 , we arrive at

$$
\left(L-\frac{\partial}{\partial t}\right)\left(\frac{|\nabla u|^{2}}{u}\right) \geq \frac{u}{n}|\Delta(\log u)|^{2}+\frac{1}{u}(\operatorname{Ric}-2 \operatorname{Hess} V)(\nabla u, \nabla u) .
$$

Theorem 2.3. Assume that there is a $k>0$ such that

$$
\text { Ric }-2 \operatorname{Hess} V \geq k \text { Id. }
$$

Then for all $t>0$,

$$
\frac{\mathrm{d}}{\mathrm{d} t} \operatorname{Ent}\left(u_{t}\right) \leq \frac{e^{-k t}}{2} \int_{M} \frac{|\nabla f|^{2}}{f} \mathrm{~d} \mu .
$$

Proof. Integrating both sides of (2.14), we obtain

$$
\int_{M}\left(L-\frac{\partial}{\partial t}\right)\left(\frac{|\nabla u|^{2}}{u}\right) \mathrm{d} \mu \geq \frac{1}{n} \int_{M} u|\Delta(\log u)|^{2} \mathrm{~d} \mu+k \int_{M} \frac{|\nabla u|^{2}}{u} \mathrm{~d} \mu .
$$

By (2.10), we have

$$
\int_{M} L\left(\frac{|\nabla u|^{2}}{u}\right) \mathrm{d} \mu=0
$$

thus the above inequality reduces to

$$
-\int_{M} \frac{\partial}{\partial t}\left(\frac{|\nabla u|^{2}}{u}\right) \mathrm{d} \mu \geq \frac{1}{n} \int_{M} u|\Delta(\log u)|^{2} \mathrm{~d} \mu+k \int_{M} \frac{|\nabla u|^{2}}{u} \mathrm{~d} \mu .
$$

Therefore

$$
\frac{\mathrm{d}}{\mathrm{d} t} \int_{M} \frac{|\nabla u|^{2}}{u} \mathrm{~d} \mu \leq-k \int_{M} \frac{|\nabla u|^{2}}{u} \mathrm{~d} \mu
$$

from which the desired estimate follows.

Now we can prove Theorem 1.1, the case when $V=0$.

Proof of Theorem 1.1. Remark that now $\mathrm{d} \mu=\mathrm{d} x$, i.e. the volume measure. We still have (2.15), or

$$
-\frac{\mathrm{d}}{\mathrm{d} t} \int_{M} \frac{|\nabla u|^{2}}{u} \mathrm{~d} x \geq \frac{1}{n} \int_{M} u|\Delta(\log u)|^{2} \mathrm{~d} x+k \int_{M} \frac{|\nabla u|^{2}}{u} \mathrm{~d} x .
$$

For simplicity of notation, we denote by $q_{t}=\int_{M} \frac{\left|\nabla u_{t}\right|^{2}}{u_{t}} \mathrm{~d} x$. Then the above inequality can be written as

$$
-\frac{\mathrm{d} q_{t}}{\mathrm{~d} t} \geq \frac{1}{n} \int_{M} u|\Delta(\log u)|^{2} \mathrm{~d} x+k q_{t} .
$$

By the Cauchy inequality,

$$
\left(\int_{M} u \Delta \log u \mathrm{~d} x\right)^{2} \leq \int_{M} u|\Delta \log u|^{2} \mathrm{~d} x \cdot \int_{M} u \mathrm{~d} x=\int_{M} u|\Delta \log u|^{2} \mathrm{~d} x .
$$

Using the integration by parts formula, we have

$$
\int_{M} u \Delta \log u \mathrm{~d} x=-\int_{M}\langle\nabla u, \nabla \log u\rangle \mathrm{d} x=-q_{t} .
$$

Therefore (2.16) becomes

$$
\frac{\mathrm{d} q_{t}}{\mathrm{~d} t} \leq-\frac{q_{t}^{2}}{n}-k q_{t} .
$$


Now we solve the above differential inequality. If $k=0$, then

$$
\frac{\mathrm{d} q_{t}}{\mathrm{~d} t} \leq-\frac{q_{t}^{2}}{n}
$$

from which we easily obtain that $q_{t} \leq \frac{n q_{0}}{n+q_{0} t}$. In the case $k \neq 0$, we divide both sides of (2.18) by $q_{t}^{2}$ and get

$$
\frac{1}{q_{t}^{2}} \frac{\mathrm{d} q_{t}}{\mathrm{~d} t} \leq-\frac{1}{n}-\frac{k}{q_{t}}
$$

This is equivalent to

$$
\frac{\mathrm{d} q_{t}^{-1}}{\mathrm{~d} t} \geq \frac{1}{n}+k q_{t}^{-1}
$$

Therefore

$$
\frac{\mathrm{d}}{\mathrm{d} t}\left(e^{-k t} q_{t}^{-1}\right) \geq \frac{e^{-k t}}{n} .
$$

Integrating this inequality from 0 to $t$ leads to

$$
e^{-k t} q_{t}^{-1}-q_{0}^{-1} \geq \frac{1}{n k}\left(1-e^{-k t}\right)
$$

The proof is now completed.

We have the following simple observations.

Corollary 2.4. Assume the conditions of Theorem 1.1.

(i) If $k>0$, then the time derivative of the entropy has an exponential decay as $t \rightarrow \infty$;

(ii) if $k<0$, then the time derivative of the entropy is asymptotically dominated by $-n k / 2$ as $t \rightarrow \infty$.

Proof. The assertions follow directly from Theorem 1.1.

We can also obtain an estimate on the rate of change of entropy from Hamilton's gradient estimate for heat equations (see [4] or [1, Corollary 3.3]).

Proposition 2.5. Let $u$ be a solution to the standard heat equation (1.1). Assume $\int_{M} f \mathrm{~d} x=1$ and there is $k \in \mathbb{R}$ such that $\mathrm{Ric} \geq k \mathrm{Id}$. Then for all $t>0$,

$$
\frac{\mathrm{d}}{\mathrm{d} t} \operatorname{Ent}\left(u_{t}\right) \leq\left(\frac{1}{t}-k\right) \log (\sup f) .
$$

Moreover, if $\mathrm{Ric} \geq 0$, then we can take $k=0$ in the above estimate.

Proof. By [1, Corollary 3.3], we have

$$
\frac{\left|\nabla u_{t}\right|^{2}}{u_{t}^{2}} \leq 2\left(\frac{1}{t}-k\right) \log \frac{A}{u_{t}},
$$

where $A=\sup _{M \times[0, t]} u=\sup _{M} f$. Therefore

$$
\frac{\left|\nabla u_{t}\right|^{2}}{u_{t}} \leq 2\left(\frac{1}{t}-k\right) u_{t} \log \frac{\sup f}{u_{t}} .
$$


Integrating both sides on $M$, we get

$$
\begin{aligned}
\int_{M} \frac{\left|\nabla u_{t}\right|^{2}}{u_{t}} \mathrm{~d} x & \leq 2\left(\frac{1}{t}-k\right) \int_{M} u_{t} \log \frac{\sup f}{u_{t}} \mathrm{~d} x \\
& =2\left(\frac{1}{t}-k\right)\left(\log (\sup f)+\operatorname{Ent}\left(u_{t}\right)\right) \leq 2\left(\frac{1}{t}-k\right) \log (\sup f),
\end{aligned}
$$

since the entropy $\operatorname{Ent}\left(u_{t}\right) \leq 0$. The proof is complete.

It is interesting to compare the two estimates given in Theorem 1.1 and Proposition 2.5. We distinguish three cases:

(a) $k<0$. By Theorem 1.1, $\frac{\mathrm{d}}{\mathrm{d} t} \operatorname{Ent}\left(u_{t}\right)$ is asymptotically dominated by $-n k / 2$, depending only on the dimension and curvature; while by Proposition 2.5, the asymptotic constant is $-k \log (\sup f)$ which depends on the initial condition.

(b) $k=0$. By Theorem 1.1, $\frac{\mathrm{d}}{\mathrm{d} t} \operatorname{Ent}\left(u_{t}\right) \leq \frac{n q_{0}}{2\left(n+q_{0} t\right)} \leq \frac{n}{2 t}$. The estimate given by Proposition 2.5 is $\frac{\mathrm{d}}{\mathrm{d} t} \operatorname{Ent}\left(u_{t}\right) \leq \frac{1}{t} \log (\sup f)$.

(c) $k>0$. Theorem 1.1 gives us an exponential decay: $\frac{\mathrm{d}}{\mathrm{d} t} \operatorname{Ent}\left(u_{t}\right) \leq \frac{1}{2} q_{0} e^{-k t}$; while Proposition 2.5 only leads to a polynomial decay: $\frac{\mathrm{d}}{\mathrm{d} t} \operatorname{Ent}\left(u_{t}\right) \leq \frac{1}{t} \log (\sup f)$.

To complete this section, we briefly discuss the entropy of the heat kernel on a non-compact Riemannian manifold with nonnegative Ricci curvature. As mentioned in the Introduction (see also [8, p.90]), the integration by parts formula can be justified rigorously, thanks to Li-Yau's gradient estimate [6]. Therefore if $u_{t}$ is the heat kernel of $M$, in the same way we can show that $\frac{\mathrm{d}}{\mathrm{d} t} \operatorname{Ent}\left(u_{t}\right) \leq \frac{n}{2 t}$. The next theorem is an analogue of [8, Theorem 1.4], which says that if the Ricci curvature is nonnegative, then the decay rate of $\frac{n}{2 t}$ is achieved if and only if $M=\mathbb{R}^{n}$.

Theorem 2.6. Let $M$ be a non-compact Riemannian manifold with nonnegative Ricci curvature, and $u_{t}$ its heat kernel. Then

$$
\frac{\mathrm{d}}{\mathrm{d} t} \operatorname{Ent}\left(u_{t}\right)=\frac{n}{2 t}
$$

if and only if $M$ is isometric to $\mathbb{R}^{n}$.

Proof. We only have to prove the necessity part. Suppose (2.20) holds. Since $M$ has nonnegative Ricci curvature, it is stochastically complete, that is, $\int_{M} u_{t} \mathrm{~d} x=1$ for all $t>0$ (see e.g. [5, Theorem 4.2.4]). By the proof of Theorem 1.1 (remark that now $k=0$ ), we must have equalities in (2.13) and (2.17). The equality in (2.13) will imply that $\Delta\left(\log u_{t}\right)=n g_{t}$ for some function $g: \mathbb{R}_{+} \times M \rightarrow \mathbb{R}$. Next the equality in (2.17) means that $g_{t}$ is a function independent of $x \in M$. By the integration by parts formula,

$$
n g_{t}=\int_{M} u_{t} \Delta\left(\log u_{t}\right) \mathrm{d} x=-\int_{M} \frac{\left|\nabla u_{t}\right|^{2}}{u_{t}} \mathrm{~d} x=-2 \frac{\mathrm{d}}{\mathrm{d} t} \operatorname{Ent}\left(u_{t}\right)=-\frac{n}{t},
$$

therefore $g_{t}=-\frac{1}{t}$ and $\Delta\left(\log u_{t}\right)=-\frac{n}{t}$.

The rest of the proof is similar to that of [8, Theorem 1.4]. By Varadhan's large deviation formula (see [5, Theorem 5.2.1]),

$$
-2 \lim _{t \rightarrow 0} t \log u_{t}(x, y)=d^{2}(x, y),
$$


where $d: M \times M \rightarrow \mathbb{R}_{+}$is the Riemannian distance function. Thus,

$$
\Delta d^{2}(x, y)=-2 \lim _{t \rightarrow 0} t \Delta \log u_{t}(x, y)=2 n .
$$

From (2.21) we deduce that

$$
\frac{A_{x}(r)}{V_{x}(r)}=n,
$$

where $A_{x}(r)$ and $V_{x}(r)$ denote respectively the area of $\partial B_{x}(r)$ and the volume of $B_{x}(r)=\{y \in$ $M: d(x, y) \leq r\}$. This implies that $V_{x}(r)$ is the same as the volume function of Euclidean balls. The equality case of the volume comparison theorem gives us $M=\mathbb{R}^{n}$.

\section{Rate of change of the entropy in terms of the spectral gap}

In this section we will obtain an estimate on the time derivative of the entropy in terms of the spectral gap. For simplicity, set $V=0$. Then we have the Sturm-Liouville decomposition of the fundamental solution,

$$
\Phi(t, x, y)=\sum_{j=0}^{\infty} e^{-\lambda_{j} t / 2} \phi_{j}(x) \phi_{j}(y)
$$

whereby $\phi_{j}$ are the eigenfunctions of $\Delta$ with eigenvalue $\lambda_{j}, 0=\lambda_{0}<\lambda_{1}<\ldots, \lambda_{j} \nearrow \infty$. In particular, each eigenvalue has finite multiplicity and each $\phi_{j}$ is smooth. Furthermore, $\left\{\phi_{j}\right\}_{j=0}^{\infty}$ form an orthonormal basis for the $L^{2}(M)$ and $\phi_{0}=1$ if $\int_{M} \Phi_{t} \mathrm{~d} y=1$, see [2, p.139]. The series in (3.1) converges absolutely and uniformly.

Given any solution $u$ to the heat equation with initial data $f$, we can write

$$
u_{t}(x)=\int_{M} f(y) \Phi(t, x, y) \mathrm{d} y=\sum_{j=0}^{\infty} e^{-\lambda_{j} t / 2} c_{j} \phi_{j}(x), c_{j}=\int_{M} f(y) \phi_{j}(y) \mathrm{d} y .
$$

And

$$
\frac{\partial}{\partial t} u_{t}(x)=\frac{1}{2} \Delta u_{t}(x)=-\sum_{j=0}^{\infty} e^{-\lambda_{j} t / 2} \lambda_{j} c_{j} \phi_{j}(x) / 2 .
$$

The series in (3.2) and (3.3) converge absolutely and uniformly for $t>0$.

Write $\langle f, g\rangle=\int_{M} f g \mathrm{~d} x$ and $\|f\|_{2}^{2}=\langle f, f\rangle$.

Proposition 3.1. Assume $M$ is a closed Riemannian manifold. Let $f \in C^{2}(M,(0, \infty))$ and $u_{t}$ be the solution to the heat equation with $u_{0}=f$. Then

$$
0 \leq \frac{\mathrm{d}}{\mathrm{d} t} \operatorname{Ent}\left(u_{t}\right) \leq \frac{1}{2} e^{-\lambda_{1} t / 2}\|\Delta f\|_{2} \sqrt{\operatorname{Vol}(M)}(|\log \inf f|+|\log \sup f|) .
$$

Here, $\operatorname{Vol}(M)$ is the volume of the manifold.

Proof. By our assumption, $\Delta f$ is continuous. Thus,

$$
\Delta f=\sum_{j=0}^{\infty}\left\langle\Delta f, \phi_{j}\right\rangle \phi_{j}=-\sum_{j=0}^{\infty} \lambda_{j}\left\langle f, \phi_{j}\right\rangle \phi_{j}=-\sum_{j=1} \lambda_{j} c_{j} \phi_{j}
$$

and $\|\Delta f\|_{2}^{2}=\langle\Delta f, \Delta f\rangle=\sum_{j=1} \lambda_{j}^{2} c_{j}^{2}<\infty$. By (3.3),

$$
\left\|\Delta u_{t}\right\|_{2}^{2}=\sum_{j=1}^{\infty} e^{-\lambda_{j} t} \lambda_{j}^{2} c_{j}^{2} \leq e^{-\lambda_{1} t} \sum_{j=1}^{\infty} \lambda_{j}^{2} c_{j}^{2}=e^{-\lambda_{1} t}\|\Delta f\|_{2}^{2} .
$$


Next by (3.2), it is clear that

$$
\int_{M}|\log u|^{2} \mathrm{~d} x \leq \operatorname{Vol}(M)(|\log \inf f|+|\log \sup f|)^{2} .
$$

Now the estimate follows from Cauchy's inequality:

$$
\begin{aligned}
\frac{\mathrm{d}}{\mathrm{d} t} \operatorname{Ent}\left(u_{t}\right) & =-\frac{1}{2} \int_{M} \log u_{t} \cdot \Delta u_{t} \mathrm{~d} \nu \\
& \leq \frac{1}{2}\left\|\log u_{t}\right\|_{2}\left\|\Delta u_{t}\right\|_{2} \\
& \leq \frac{1}{2} e^{-\lambda_{1} t / 2}\|\Delta f\|_{2} \sqrt{\operatorname{Vol}(M)}(|\log \inf f|+|\log \sup f|) .
\end{aligned}
$$

\section{Example: Entropy of the heat kernel on the three dimensional hyperbolic space $\mathbb{H}^{3}$}

In this section we consider the three dimensional hyperbolic space $\mathbb{H}^{3}$ of constant sectional curvature $k<0$. Let $d(\cdot, \cdot)$ be the Riemannian distance function on $\mathbb{H}^{3}$. The heat kernel on $\mathbb{H}^{3}$ has the following explicit formula (see $[2, \mathrm{p} .150])$ :

$$
h(t, x, y)=e^{-d(x, y)^{2} / 2 t}(2 \pi t)^{-3 / 2} \frac{\sqrt{-k} d(x, y)}{\sinh \sqrt{-k} d(x, y)} e^{k t / 2}, \quad x, y \in \mathbb{H}^{3} .
$$

(There is a mistake in the formula for $h(t, x, y)$ given in [5, Example 5.1.3]: there the last factor is $e^{-t}(k=-1)$, rather than $e^{-t / 2}$.) Let $\mathrm{V}_{\mathbb{H}^{3}}$ be the volume measure on $\mathbb{H}^{3}$ and define the entropy

$$
\operatorname{Ent}(h(t, x, \cdot))=-\int_{\mathbb{H}^{3}} h(t, x, y) \log h(t, x, y) \mathrm{dV}_{\mathbb{H}^{3}}(y), \quad(t, x) \in(0, \infty) \times \mathbb{H}^{3} .
$$

The main result in this section is

\section{Theorem 4.1.}

$$
-k(2-\log \sqrt{2}) \leq \varliminf_{t \rightarrow \infty} \frac{\mathrm{d}}{\mathrm{d} t} \operatorname{Ent}(h(t, x, \cdot)) \leq \varlimsup_{t \rightarrow \infty} \frac{\mathrm{d}}{\mathrm{d} t} \operatorname{Ent}(h(t, x, \cdot)) \leq-k(2+\log \sqrt{2}) .
$$

To prove this theorem, we need some preparations. We denote by $\kappa=\sqrt{-k}$ for the simplification of notations. Using polar coordinates the metric on $\mathbb{H}^{3}$ is expressed as

$$
\mathrm{d} s^{2}=\mathrm{d} r^{2}+\kappa^{-2}(\sinh \kappa r)^{2} \mathrm{~d} \theta^{2},
$$

where $\mathrm{d} \theta$ is the standard volume measure on the sphere $S^{2}$. Hence for any integrable function $f: \mathbb{H}^{3} \rightarrow \mathbb{R}$, the following equality holds:

$$
\int_{\mathbb{H}^{3}} f(x) \mathrm{dV}_{\mathbb{H}^{3}}(x)=\int_{0}^{\infty}\left(\int_{S^{2}} f(r \theta) \mathrm{d} \theta\right) \frac{(\sinh \kappa r)^{2}}{\kappa^{2}} \mathrm{~d} r .
$$

We have

$$
\operatorname{Ent}(h(t, x, \cdot))=\frac{3}{2} \log (2 \pi t)+\frac{\kappa^{2} t}{2}+I_{1}(t)+I_{2}(t)
$$


where

$$
\begin{aligned}
& I_{1}(t)=\frac{1}{2 t} \int_{\mathbb{H}^{3}} h(t, x, y) d(x, y)^{2} \mathrm{dV}_{\mathbb{H}^{3}}(y), \\
& I_{2}(t)=\int_{\mathbb{H}^{3}} h(t, x, y) \log \frac{\sinh \kappa d(x, y)}{\kappa d(x, y)} \mathrm{dV}_{\mathbb{H}^{3}}(y) .
\end{aligned}
$$

We first compute $I_{1}(t)$. Using the expression of $h(t, x, y)$ and by the formula (4.1), we have

$$
\begin{aligned}
I_{1}(t) & =\frac{1}{2 t(2 \pi t)^{3 / 2} e^{\kappa^{2} t / 2}} \int_{0}^{\infty}\left(\int_{S^{2}} e^{-r^{2} / 2 t} \frac{\kappa r}{\sinh \kappa r} r^{2} \mathrm{~d} \theta\right) \frac{(\sinh \kappa r)^{2}}{\kappa^{2}} \mathrm{~d} r \\
& =\frac{1}{\sqrt{2 \pi} \kappa t^{5 / 2} e^{\kappa^{2} t / 2}} I_{11}(t),
\end{aligned}
$$

where $I_{11}(t)=\int_{0}^{\infty} e^{-r^{2} / 2 t} r^{3} \sinh \kappa r \mathrm{~d} r$. Here we collect some results for later use.

Lemma 4.2. Let $\alpha(t)=\int_{0}^{\kappa t^{1 / 2}} e^{-r^{2} / 2} \mathrm{~d} r$. Then

$$
\begin{aligned}
\int_{0}^{\infty} e^{-r^{2} / 2 t} \sinh \kappa r \mathrm{~d} r & =t^{1 / 2} e^{\kappa^{2} t / 2} \alpha(t), \\
\int_{0}^{\infty} e^{-r^{2} / 2 t} \cosh \kappa r \mathrm{~d} r & =\sqrt{\frac{\pi}{2}} t^{1 / 2} e^{\kappa^{2} t / 2}, \\
\int_{0}^{\infty} e^{-r^{2} / 2 t} r \sinh \kappa r \mathrm{~d} r & =\sqrt{\frac{\pi}{2}} \kappa t^{3 / 2} e^{\kappa^{2} t / 2}, \\
\int_{0}^{\infty} e^{-r^{2} / 2 t} r \cosh \kappa r \mathrm{~d} r & =t+\kappa t^{3 / 2} e^{\kappa^{2} t / 2} \alpha(t), \\
\int_{0}^{\infty} e^{-r^{2} / 2 t} r^{2} \sinh \kappa r \mathrm{~d} r & =\kappa t^{2}+t^{3 / 2}\left(\kappa^{2} t+1\right) e^{\kappa^{2} t / 2} \alpha(t), \\
\int_{0}^{\infty} e^{-r^{2} / 2 t} r^{2} \cosh \kappa r \mathrm{~d} r & =\sqrt{\frac{\pi}{2}} t^{3 / 2}\left(\kappa^{2} t+1\right) e^{\kappa^{2} t / 2}, \\
\int_{0}^{\infty} e^{-r^{2} / 2 t} r^{3} \sinh \kappa r \mathrm{~d} r & =\sqrt{\frac{\pi}{2}} \kappa t^{5 / 2}\left(\kappa^{2} t+3\right) e^{\kappa^{2} t / 2}, \\
\int_{0}^{\infty} e^{-r^{2} / 2 t} r^{3} \cosh \kappa r \mathrm{~d} r & =t^{2}\left(\kappa^{2} t+2\right)+\kappa t^{5 / 2}\left(\kappa^{2} t+3\right) e^{\kappa^{2} t / 2} \alpha(t), \\
\int_{0}^{\infty} e^{-r^{2} / 2 t} r^{4} \sinh \kappa r \mathrm{~d} r & =\kappa t^{3}\left(\kappa^{2} t+5\right)+t^{5 / 2}\left(\kappa^{4} t^{2}+6 \kappa^{2} t+3\right) e^{\kappa^{2} t / 2} \alpha(t) .
\end{aligned}
$$

Proof. We only prove the first two equalities. The others can be proved using the integration by parts formula. We have

$$
\int_{0}^{\infty} e^{-r^{2} / 2 t} e^{\kappa r} \mathrm{~d} r=e^{\kappa^{2} t / 2} \int_{-\kappa t}^{\infty} e^{-r^{2} / 2 t} \mathrm{~d} r=t^{1 / 2} e^{\kappa^{2} t / 2} \int_{-\kappa t^{1 / 2}}^{\infty} e^{-r^{2} / 2} \mathrm{~d} r
$$

and

$$
\int_{0}^{\infty} e^{-r^{2} / 2 t} e^{-\kappa r} \mathrm{~d} r=e^{\kappa^{2} t / 2} \int_{\kappa t}^{\infty} e^{-r^{2} / 2 t} \mathrm{~d} r=t^{1 / 2} e^{\kappa^{2} t / 2} \int_{\kappa t^{1 / 2}}^{\infty} e^{-r^{2} / 2} \mathrm{~d} r
$$

Hence

$$
\int_{0}^{\infty} e^{-r^{2} / 2 t} \sinh r \mathrm{~d} r=\frac{1}{2} t^{1 / 2} e^{\kappa^{2} t / 2} \int_{-\kappa t^{1 / 2}}^{\kappa t^{1 / 2}} e^{-r^{2} / 2} \mathrm{~d} r=t^{1 / 2} e^{\kappa^{2} t / 2} \alpha(t) .
$$


Next since the function $r \mapsto \cosh \kappa r$ is even,

$$
\begin{aligned}
\int_{0}^{\infty} e^{-r^{2} / 2 t} \cosh \kappa r \mathrm{~d} r & =\frac{1}{2} \int_{-\infty}^{\infty} e^{-r^{2} / 2 t} \cosh r \mathrm{~d} r \\
& =\frac{1}{2} \int_{-\infty}^{\infty} e^{-r^{2} / 2 t} e^{\kappa r} \mathrm{~d} r=\sqrt{\frac{\pi}{2}} t^{1 / 2} e^{\kappa^{2} t / 2}
\end{aligned}
$$

By Lemma 4.2, we obtain

$$
I_{1}(t)=\frac{1}{\sqrt{2 \pi} \kappa t^{5 / 2} e^{\kappa^{2} t / 2}} \sqrt{\frac{\pi}{2}} \kappa t^{5 / 2}\left(\kappa^{2} t+3\right) e^{\kappa^{2} t / 2}=\frac{1}{2}\left(\kappa^{2} t+3\right) .
$$

Now we consider $I_{2}(t)$. Again by (4.1),

$$
I_{2}(t)=\sqrt{\frac{2}{\pi}} \kappa^{-1} t^{-3 / 2} e^{-\kappa^{2} t / 2} \int_{0}^{\infty} e^{-2 r^{2} / 2 t} r \sinh \kappa r \log \frac{\sinh \kappa r}{\kappa r} \mathrm{~d} r .
$$

Due to the presence of the term $\log \frac{\sinh \kappa r}{\kappa r}$, we are unable to compute $I_{2}(t)$ explicitly. In the sequel we intend to find some estimates on it. Define

$$
\xi(t)=\sqrt{\frac{2}{\pi}} \kappa^{-1} t^{-3 / 2} e^{-\kappa^{2} t / 2} \quad \text { and } \quad \eta(t)=\int_{0}^{\infty} e^{-r^{2} / 2 t} r \sinh \kappa r \log \frac{\sinh \kappa r}{\kappa r} \mathrm{~d} r .
$$

We have

$$
\xi^{\prime}(t)=-\frac{1}{\sqrt{2 \pi} \kappa} \cdot \frac{\kappa^{2} t+3}{t^{5 / 2} e^{\kappa^{2} t / 2}}<0 .
$$

Therefore, to estimate $I_{2}^{\prime}(t)$, it is enough to estimate $\eta(t)$ and $\eta^{\prime}(t)$.

The following lemma gives the key ingredient.

Lemma 4.3. For any $r>0$,

$$
\frac{1}{1+2 r}<\frac{1-e^{-2 r}}{2 r}<\frac{1}{1+r}
$$

Proof. (1) Let $\phi(s)=1-e^{-s}-s e^{-s}$. Then $\phi(0)=0$ and $\phi^{\prime}(s)=s e^{-s}>0$. Hence $\phi(s)>0$ for all $s>0$. This implies

$$
1+s-e^{-s}-s e^{-s}>s,
$$

which leads to the first inequality by taking $s=2 r$.

(2) Let $\psi(r)=1-r-e^{-2 r}-r e^{-2 r}$. Then $\psi(0)=0$ and $\psi^{\prime}(r)=-1+e^{-2 r}+2 r e^{-2 r}$. We have $\psi^{\prime}(0)=0$ and $\psi^{\prime \prime}(r)=-4 r e^{-2 r}<0$ for any $r>0$. Therefore $\psi^{\prime}(r)<0$. As a result, $\psi(r)<0$ for all $r>0$. This implies that

$$
1+r-e^{-2 r}-r e^{-2 r}<2 r
$$

which is equivalent to the second inequality.

Remark 4.4. Fix any $\beta \in(1,2)$. In the same way we can show that for $r \in\left(0, \frac{\beta-1}{\beta}\right)$, it holds $\frac{1-e^{-2 r}}{2 r}>\frac{1}{1+\beta r}$. Moreover, it is clear that $\frac{1-e^{-2 r}}{2 r}<\frac{1}{1+\beta r}$ when $r$ is sufficiently large. Therefore the two inequalities in Lemma 4.3 are sharp in this sense. 
Notice that

$$
\frac{\sinh \kappa r}{\kappa r}=\frac{e^{\kappa r}\left(1-e^{-2 \kappa r}\right)}{2 \kappa r}
$$

thus by Lemma 4.3,

$$
\kappa r+\log \frac{1}{1+2 \kappa r}<\log \frac{\sinh \kappa r}{\kappa r}<\kappa r+\log \frac{1}{1+\kappa r}, \quad r>0 .
$$

Now we can obtain the upper and lower bound on $\eta(t)$.

\section{Lemma 4.5.}

$$
\begin{aligned}
& \eta(t)>\kappa^{2} t^{2}+\kappa t^{3 / 2}\left(\kappa^{2} t+1\right) e^{\kappa^{2} t / 2} \alpha(t)-\sqrt{\frac{\pi}{2}} \kappa t^{3 / 2} e^{\kappa^{2} t / 2} \log \left(2 \kappa^{2} t+4\right) \\
& \eta(t)<\kappa^{2} t^{2}+\kappa t^{3 / 2}\left(\kappa^{2} t+1\right) e^{\kappa^{2} t / 2} \alpha(t)-\sqrt{\frac{\pi}{2}} \kappa t^{3 / 2} e^{\kappa^{2} t / 2} \log \left(1+\sqrt{\frac{\pi}{2}} \kappa^{2} t \alpha(t)^{-1}\right) .
\end{aligned}
$$

Proof. By the definition (4.7) and the inequality (4.9), we have

$$
\eta(t)>\eta_{1}(t)-\eta_{2}(t)
$$

where by Lemma 4.2 ,

$$
\eta_{1}(t)=\kappa \int_{0}^{\infty} e^{-r^{2} / 2 t} r^{2} \sinh \kappa r \mathrm{~d} r=\kappa^{2} t^{2}+\kappa t^{3 / 2}\left(\kappa^{2} t+1\right) e^{\kappa^{2} t / 2} \alpha(t)
$$

and $\eta_{2}(t)=\int_{0}^{\infty} e^{-r^{2} / 2 t} r \log (1+2 \kappa r) \sinh \kappa r \mathrm{~d} r$. Define the measure

$$
\mathrm{d} \mu(r)=\sqrt{\frac{2}{\pi}} \kappa^{-1} t^{-3 / 2} e^{-\kappa^{2} t / 2} e^{-r^{2} / 2 t} r \sinh \kappa r \mathrm{~d} r
$$

on $[0, \infty)$. By Lemma $4.2, \mu$ is a probability. Notice that the function $r \mapsto \log (1+2 \kappa r)$ is concave, by Jensen's inequality,

$$
\eta_{2}(t)=\sqrt{\frac{\pi}{2}} \kappa t^{3 / 2} e^{\kappa^{2} t / 2} \int_{0}^{\infty} \log (1+2 \kappa r) \mathrm{d} \mu(r) \leq \sqrt{\frac{\pi}{2}} \kappa t^{3 / 2} e^{\kappa^{2} t / 2} \log \left(1+2 \kappa \int_{0}^{\infty} r \mathrm{~d} \mu(r)\right) .
$$

Again by Lemma 4.2,

$$
\begin{aligned}
\int_{0}^{\infty} r \mathrm{~d} \mu(r) & =\sqrt{\frac{2}{\pi}} \kappa^{-1} t^{-3 / 2} e^{-\kappa^{2} t / 2} \int_{0}^{\infty} e^{-r^{2} / 2 t} r^{2} \sinh \kappa r \mathrm{~d} r \\
& =\sqrt{\frac{2}{\pi}} t^{1 / 2} e^{-\kappa^{2} t / 2}+\sqrt{\frac{2}{\pi}} \kappa^{-1}\left(\kappa^{2} t+1\right) \alpha(t) .
\end{aligned}
$$

It is easy to show that $\sqrt{\frac{2}{\pi}} t^{1 / 2} e^{-\kappa^{2} t / 2} \leq \frac{1}{\kappa} \sqrt{\frac{2}{\pi e}} \leq \frac{1}{2 \kappa}$ and $\alpha(t) \leq \sqrt{\frac{\pi}{2}}$. Hence

$$
\int_{0}^{\infty} r \mathrm{~d} \mu(r) \leq \frac{1}{2 \kappa}+\kappa t+\frac{1}{\kappa}=\kappa t+\frac{3}{2 \kappa}
$$

Consequently

$$
\eta_{2}(t) \leq \sqrt{\frac{\pi}{2}} \kappa t^{3 / 2} e^{\kappa^{2} t / 2} \log \left(2 \kappa^{2} t+4\right) .
$$

Combining this with (4.10) and (4.11), we obtain the first inequality. 
Now we prove the second inequality. By (4.9), we have

$$
\eta(t)<\eta_{1}(t)-\bar{\eta}_{2}(t)
$$

where $\eta_{1}(t)$ is defined in (4.11) and

$$
\bar{\eta}_{2}(t)=\int_{0}^{\infty} e^{-r^{2} / 2 t} r \log (1+\kappa r) \sinh \kappa r \mathrm{~d} r .
$$

Define the measure

$$
\mathrm{d} \nu(r)=t^{-1 / 2} e^{-\kappa^{2} t / 2} \alpha(t)^{-1} e^{-r^{2} / 2 t} \sinh \kappa r \mathrm{~d} r
$$

on $[0, \infty)$. Then by Lemma $4.2, \nu$ is also a probability. Notice that the function $r \mapsto r \log (1+\kappa r)$ is convex on $[0, \infty)$, again by Jensen's inequality,

$$
\begin{aligned}
\bar{\eta}_{2}(t) & =t^{1 / 2} e^{\kappa^{2} t / 2} \alpha(t) \int_{0}^{\infty} r \log (1+\kappa r) \mathrm{d} \nu(r) \\
& \geq t^{1 / 2} e^{\kappa^{2} t / 2} \alpha(t)\left(\int_{0}^{\infty} r \mathrm{~d} \nu(r)\right) \log \left(1+\kappa \int_{0}^{\infty} r \mathrm{~d} \nu(r)\right) .
\end{aligned}
$$

By Lemma 4.2, we have

$$
\int_{0}^{\infty} r \mathrm{~d} \nu(r)=t^{-1 / 2} e^{-\kappa^{2} t / 2} \alpha(t)^{-1} \int_{0}^{\infty} e^{-r^{2} / 2 t} r \sinh \kappa r \mathrm{~d} r=\sqrt{\frac{\pi}{2}} \kappa t \alpha(t)^{-1} .
$$

Therefore

$$
\bar{\eta}_{2}(t) \geq \sqrt{\frac{\pi}{2}} \kappa t^{3 / 2} e^{\kappa^{2} t / 2} \log \left(1+\sqrt{\frac{\pi}{2}} \kappa^{2} t \alpha(t)^{-1}\right) .
$$

Combining this with (4.12) and (4.11) gives the second inequality.

Note that

$$
\eta^{\prime}(t)=\frac{1}{2 t^{2}} \int_{0}^{\infty} e^{-r^{2} / 2 t} r^{3} \sinh \kappa r \log \frac{\sinh \kappa r}{\kappa r} \mathrm{~d} r .
$$

In the same way we can prove the bounds on $\eta^{\prime}(t)$.

\section{Lemma 4.6.}

$$
\begin{aligned}
\eta^{\prime}(t)> & \frac{1}{2} \kappa^{2} t\left(\kappa^{2} t+5\right)+\frac{1}{2} \kappa t^{1 / 2}\left(\kappa^{4} t^{2}+6 \kappa^{2} t+3\right) e^{\kappa^{2} t / 2} \alpha(t) \\
- & \frac{1}{2} \sqrt{\frac{\pi}{2}} \kappa t^{1 / 2}\left(\kappa^{2} t+3\right) e^{\kappa^{2} t / 2} \\
& \quad \times \log \left(1+2 \kappa \sqrt{\frac{2}{\pi}} \frac{t^{1 / 2}\left(\kappa^{2} t+5\right)}{\kappa^{2} t+3} e^{-\kappa^{2} t / 2}+2 \sqrt{\frac{2}{\pi}} \frac{\kappa^{4} t^{2}+6 \kappa^{2} t+3}{\kappa^{2} t+3} \alpha(t)\right), \\
\eta^{\prime}(t)< & \frac{1}{2} \kappa^{2} t\left(\kappa^{2} t+5\right)+\frac{1}{2} \kappa t^{1 / 2}\left(\kappa^{4} t^{2}+6 \kappa^{2} t+3\right) e^{\kappa^{2} t / 2} \alpha(t) \\
- & \frac{1}{2} \sqrt{\frac{\pi}{2}} \kappa t^{1 / 2}\left(\kappa^{2} t+3\right) e^{\kappa^{2} t / 2} \log \left(1+\frac{\kappa^{2} t\left(\kappa^{2} t+3\right) e^{\kappa^{2} t / 2}}{\kappa t^{1 / 2}+\left(\kappa^{2} t+1\right) e^{\kappa^{2} t / 2} \alpha(t)} \sqrt{\frac{\pi}{2}}\right) .
\end{aligned}
$$

Proof. The proofs are similar to Lemma 4.5, hence we only give a sketch here. By (4.13) and (4.9), we have

$$
\eta^{\prime}(t)>\eta_{3}(t)-\eta_{4}(t)
$$


where, by Lemma 4.2,

$$
\begin{aligned}
\eta_{3}(t) & =\frac{\kappa}{2 t^{2}} \int_{0}^{\infty} e^{-r^{2} / 2 t} r^{4} \sinh \kappa r \mathrm{~d} r \\
& =\frac{1}{2} \kappa^{2} t\left(\kappa^{2} t+5\right)+\frac{1}{2} \kappa t^{1 / 2}\left(\kappa^{4} t^{2}+6 \kappa^{2} t+3\right) e^{\kappa^{2} t / 2} \alpha(t)
\end{aligned}
$$

and

$$
\eta_{4}(t)=\frac{1}{2 t^{2}} \int_{0}^{\infty} e^{-r^{2} / 2 t} r^{3} \log (1+2 \kappa r) \sinh \kappa r \mathrm{~d} r .
$$

As in Lemma 4.5, using the concavity of $r \mapsto \log (1+2 \kappa r)$, we can get

$$
\begin{aligned}
\eta_{4}(t) \leq & \frac{1}{2} \sqrt{\frac{\pi}{2}} \kappa t^{1 / 2}\left(\kappa^{2} t+3\right) e^{\kappa^{2} t / 2} \\
& \times \log \left(1+2 \kappa \sqrt{\frac{2}{\pi}} \frac{t^{1 / 2}\left(\kappa^{2} t+5\right)}{\kappa^{2} t+3} e^{-\kappa^{2} t / 2}+2 \sqrt{\frac{2}{\pi}} \frac{\kappa^{4} t^{2}+6 \kappa^{2} t+3}{\kappa^{2} t+3} \alpha(t)\right) .
\end{aligned}
$$

Together with (4.14) and (4.15) gives us the first inequality.

Next, by (4.9),

$$
\eta^{\prime}(t)<\eta_{3}(t)-\bar{\eta}_{4}(t)
$$

where $\eta_{3}(t)$ is defined in (4.15) and

$$
\bar{\eta}_{4}(t)=\frac{1}{2 t^{2}} \int_{0}^{\infty} e^{-r^{2} / 2 t} r^{3} \log (1+\kappa r) \sinh \kappa r \mathrm{~d} r .
$$

Using the convexity of the function $r \mapsto r \log (1+\kappa r)$, we can show that

$$
\bar{\eta}_{4}(t) \geq \frac{1}{2} \sqrt{\frac{\pi}{2}} \kappa t^{1 / 2}\left(\kappa^{2} t+3\right) e^{\kappa^{2} t / 2} \log \left(1+\frac{\kappa^{2} t\left(\kappa^{2} t+3\right) e^{\kappa^{2} t / 2}}{\kappa t^{1 / 2}+\left(\kappa^{2} t+1\right) e^{\kappa^{2} t / 2} \alpha(t)} \sqrt{\frac{\pi}{2}}\right) .
$$

Now the second inequality follows from the above estimate and (4.16), (4.15).

Finally we are ready to prove Theorem 4.1.

Proof of Theorem 4.1. First we consider the upper limit of the time derivative of the entropy. By (4.8) and Lemma 4.5, we have

$$
\xi^{\prime}(t) \eta(t)<-\frac{\kappa}{\sqrt{2 \pi}} \frac{\kappa^{2} t+3}{t^{1 / 2} e^{\kappa^{2} t / 2}}-\frac{\alpha(t)}{\sqrt{2 \pi}} \frac{\kappa^{4} t^{2}+4 \kappa^{2} t+3}{t}+\frac{\kappa^{2} t+3}{2 t} \log \left(2 \kappa^{2} t+4\right) .
$$

Recall that $\alpha(t)$ is defined in Lemma 4.2 and $\lim _{t \rightarrow \infty} \alpha(t)=\sqrt{\frac{\pi}{2}}$. And by Lemma 4.6, we have

$$
\begin{aligned}
\xi(t) \eta^{\prime}(t)< & \frac{\kappa}{\sqrt{2 \pi}} \frac{\kappa^{2} t+5}{t^{1 / 2} e^{\kappa^{2} t / 2}}+\frac{\alpha(t)}{\sqrt{2 \pi}} \frac{\kappa^{4} t^{2}+6 \kappa^{2} t+3}{t} \\
& -\frac{\kappa^{2} t+3}{2 t} \log \left(1+\frac{\kappa^{2} t\left(\kappa^{2} t+3\right) e^{\kappa^{2} t / 2}}{\kappa t^{1 / 2}+\left(\kappa^{2} t+1\right) e^{\kappa^{2} t / 2} \alpha(t)} \sqrt{\frac{\pi}{2}}\right) .
\end{aligned}
$$

Summing up the above two estimates, we obtain

$$
\frac{\mathrm{d}}{\mathrm{d} t}(\xi(t) \eta(t))<\sqrt{\frac{2}{\pi}} \frac{\kappa}{t^{1 / 2} e^{\kappa^{2} t / 2}}+\sqrt{\frac{2}{\pi}} \kappa^{2} \alpha(t)+\frac{\kappa^{2} t+3}{2 t} \log \frac{2 \kappa^{2} t+4}{1+\frac{\kappa^{2} t\left(\kappa^{2} t+3\right) e^{\kappa^{2} t / 2}}{\kappa t^{1 / 2}+\left(\kappa^{2} t+1\right) e^{\kappa^{2} t / 2} \alpha(t)} \sqrt{\frac{\pi}{2}}} .
$$


From this it is easy to see that

$$
\varlimsup_{t \rightarrow \infty} \frac{\mathrm{d}}{\mathrm{d} t}(\xi(t) \eta(t)) \leq \kappa^{2}+\frac{\kappa^{2}}{2} \log 2=\kappa^{2}(1+\log \sqrt{2}) .
$$

Similarly we have

$$
\varliminf_{t \rightarrow \infty} \frac{\mathrm{d}}{\mathrm{d} t}(\xi(t) \eta(t)) \geq \kappa^{2}-\frac{\kappa^{2}}{2} \log 2=\kappa^{2}(1-\log \sqrt{2}) .
$$

By (4.2) and (4.5)-(4.7),

$$
\frac{\mathrm{d}}{\mathrm{d} t} \operatorname{Ent}(h(t, x, \cdot))=\frac{3}{2 t}+\kappa^{2}+\frac{\mathrm{d}}{\mathrm{d} t}(\xi(t) \eta(t)),
$$

Noting that $\kappa^{2}=-k$, we finally complete the proof.

\section{References}

[1] Marc Arnaudon and Anton Thalmaier, Li-Yau type gradient estimates and harnack inequalities by stochastic analysis. Probabilistic approach to geometry, 29-48, Adv. Stud. Pure Math., 57, Math. Soc. Japan, Tokyo, 2010.

[2] Issac Chavel. Eigenvalues in Riemannian geometry, Pure and Applied Mathematics, vol. 115, Academic Press Inc., Orlando, FL, 1984.

[3] B. Chow, S.-C. Chu, D. Glickenstein, C. Guenther, J. Isenberg, T. Ivey, D. Knopf, P. Lu, F. Luo and L. Ni, The Ricci Flow: Techniques and Applications, Part II, Mathemat- ical Surveys and Monographs, AMS, Providence, RI, 2007.

[4] R. S. Hamilton, A matrix Harnack estimate for the heat equation. Comm. Anal. Geom. 1 (1993), no. 1, 113-126.

[5] Elton P. Hsu, Stochastic analysis on manifolds, Graduate Studies in Mathematics, vol. 38, American Mathematical Society, Providence, RI, 2002.

[6] P. Li and S.-T. Yau, On the parabolic kernel of the Schrödinger operator. Acta. Math. 156 (1986), 139-168.

[7] Brett Kotschwar and Lei Ni, Local gradient estimates of $p$-harmonic functions, $1 / H$-flow, and an entropy formula. Ann. Sci. c. Norm. Supr. (4) 42 (2009), no. 1, 1-36.

[8] Lei Ni, The entropy formula for linear heat equation. J. Geom. Anal. 14 (2004), no. 1, $87-100$.

[9] Lei Ni, Addenda to "The entropy formula for linear heat equation". J. Geom. Anal. 14 (2004), no. 2, 369-374. 